\title{
Dynamic Whole-Body Control of Unstable Wheeled Humanoid Robots
}

\author{
Grazia Zambella $^{1}$, Gianluca Lentini ${ }^{1,2}$, Manolo Garabini ${ }^{1}$, Giorgio Grioli ${ }^{2}$, Manuel G. Catalano ${ }^{2}$, \\ Alessandro Palleschi ${ }^{1}$, Lucia Pallottino ${ }^{1}$, Antonio Bicchi ${ }^{1,2}$, Alessandro Settimi ${ }^{1}$, Danilo Caporale ${ }^{1}$
}

\begin{abstract}
Control of two-wheeled humanoid robots poses several challenges due to the unstable dynamics of their mobile base and the coupling between upper and lower body dynamics. The problem is often faced in the literature with methods based on linearized or simplified models that fail in exploiting the whole body dynamic capabilities of the platform in use.

In this work we tackle the problem of whole-body dynamic control for a mobile wheeled unstable humanoid robot.

Compared to existing approaches based on on-line optimization to guarantee respect of the constraints, we propose a control method that takes into account the nominal constrained dynamics of the robot in the quasi-velocities through an internal model, thus reducing the computational burden. A computed-torque control law in the quasi-velocities is used to stabilize the robot around the upper position. We report on preliminary experimental results and on the method effectiveness in rejecting unknown external disturbances.
\end{abstract}

Index Terms-Wheeled Robots; Underactuated Robots; Humanoid Robots

\section{INTRODUCTION}

$\mathbf{R}$ OBOTS able to perform loco-manipulation tasks are becoming more and more popular. Several solutions have been implemented featuring different locomotion principles: see [1] for a complete survey on two-wheeled robots, [2] for ballbot robots, and the seminal work [3] for legged robots.

From the control point of view, these platforms can be classified in statically stable and unstable ones. One of the most relevant loco-manipulation control approaches suitable

Manuscript received: February, 24, 2019; Revised April, 22, 2019; Accepted June, 17, 2019.

This paper was recommended for publication by Editor Dezhen Song upon evaluation of the Associate Editor and Reviewers' comments. This work was supported in part by the European Unions Horizon 2020 research and innovation program as part of the projects ILIAD (Grant no. 732737), and in part by the Italian Ministry of Education and Research in the framework of the CrossLab project (Departments of Excellence).

1 G. Zambella, M. Garabini, A. Palleschi, L. Pallottino, A. Settimi, D. Caporale are with the Centro di ricerca E. Piaggio and the Information Engineering Department, University of Pisa, 56122 Pisa, Italy gr.zambella@gmail.com

2 G. Grioli and M. G. Catalano are with Soft Robotics for Human Cooperationand Rehabilitation, Fondazione Istituto Italiano di Tecnologia, 16163 Genova, Italy

1,2 G. Lentini and A. Bicchi are with the Centro di ricerca E. Piaggio and the Information Engineering Department, University of Pisa, 56122, Pisa, Italy, and also with Soft Robotics for Human Cooperation and Rehabilitation, Fondazione Istituto Italiano di Tecnologia, 16163 Genova, Italy.

Digital Object Identifier (DOI): see top of this page.

Authors would like to thank Gaspare Santaera, Mattia Poggiani and Michele Maimeri for their work on the ALTER-EGO software and electronics frameworks, and Cristiano Petrocelli for his work on the design and implementation of the ALTER-EGO robot.

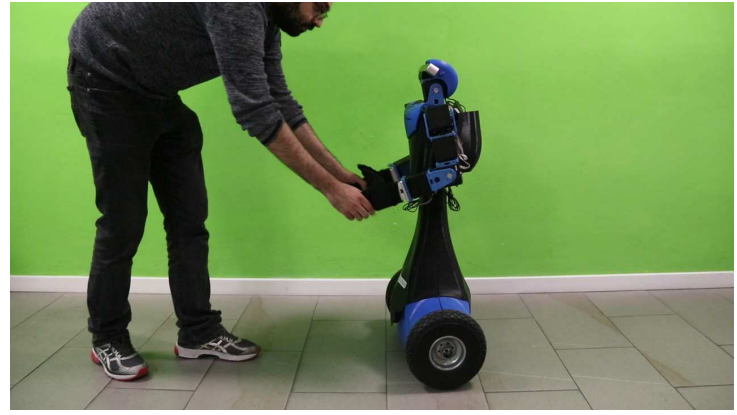

Fig. 1: ALTER-EGO - an underactuated wheeled humanoid robot.

for robots of the former type has been presented by Dietrich in [4], where the whole-body control of Rollin' Justin, a wheeled humanoid robot, is treated with an impedance method. Statically stable mobile platforms with large support base (such as Rollin' Justin) allow the control designer to focus on task accomplishment. As soon as the size of the support base is reduced, the balancing problem should also be taken into account as one of the primary control objectives. This is the case of biped robots. In [5], a quasi-static control tool for whole-body loco-manipulation is proposed. This method is used to find the contact force distribution that maximizes robot stability. In [6], a dynamic control is proposed to achieve compliant balancing for a humanoid robot utilizing multiple contacts, while ensuring the passivity of the overall system.

Unstable mobile robot may offer more mobility compared to the stable ones, at the price of a more complex control system that should first guarantee balancing and then task accomplishment.

Notice that several living beings present unstable dynamics. A prominent and surprising example is the ankle stiffness in humans that is the $80-90 \%$ of the value required for the stability of the upper equilibrium [7].

One of the main challenges when dealing with unstable platforms is the underactuation, which complicates the control design.

Control of under-actuated systems is often performed via approximate linearization about an unstable equilibrium, such as LQR [8]. Partial feedback linearization can be applied to achieve a larger region of attraction for a given equilibrium exploiting the dynamics of the system [9], although in general only external stability can be guaranteed.

Usually in wheeled humanoid robots the underactuation is at the pitch joint, it affects the balancing of the robot and 
traditionally is tackled by decoupling the control laws used to regulate the upper body and the mobile base motion. In particular, in [8], a feedback control method is proposed and applied to I-PENTAR, a wheeled inverse pendulum robot. The motion equation of this robot is computed considering the kinematic constraints on the wheeled base and an LQR balancing control is applied, tacking into account the upper body movement as a disturbance. In [10], the authors adopted a pole-placement controller to control the mobile base. In [11] a learning based method has been applied for dexterous control of a mobile manipulator (uBot-5). In [12], inspired by [13], the authors proposed a whole-body control framework for Golem Krang [14]: they applied a hierarchical approach where a Quadratic Programming low-level controller, taking as inputs the centre of Mass targets (generated and controlled by a Model Predictive high-level controller) and the task references, is used to compute the joint torques ensuring the robot balancing and locomotion, while performing other tasks with the upper body.

The contribution of the paper is a novel whole-body control for a wheeled humanoid robot (see Fig. 11). This approach aims at making the robot upper body actively cooperate with the mobile base for balancing. At the same time, our approach guarantees the respect of kinematic constraints without performing on-line optimization, as done in [12], but designing the stabilizing control law directly on the constrained dynamics of the robot.

The rest of the paper first presents the derivation of the constrained dynamics equations of the robot. Later, we show how the underactuated constrained mechanical system can be controlled with a computed torque law in the quasi-velocities. To the best of the authors' knowledge, this is the first example of the application of such approach to wheeled humanoid robots in the literature.

We demonstrate the efficacy of the proposed method with experimental validations and with simulation comparisons with state-of-the-art LQR approach on a two-wheeled unstable robot with a humanoid torso equipped with 5 degrees of freedom on each arm. The reported experiments are aimed at assessing the effectiveness of the proposed method in rejecting static and impulsive disturbances, which might affect the operation of unsupervised robots located in remote areas, or in crowded ones. Finally, we apply a task-priority approach to perform a task with unknown interaction with the environment, namely to open and close a drawer.

\section{ROBOT MODEL}

In this section we present the method focusing the discussion on the kinematic and dynamic models of a two wheeled humanoid, moving on flat plane without rolling around its sagittal axis. Let $n$ be the actuated degrees of freedom (DoF).

\section{A. Robot kinematics}

With reference to Fig. 2, we define an inertial reference frame attached to the world, $\{\mathcal{I}\}$, and a base frame $\{\mathcal{B}\}$ attached to the robot's base link. In this way, given the pose

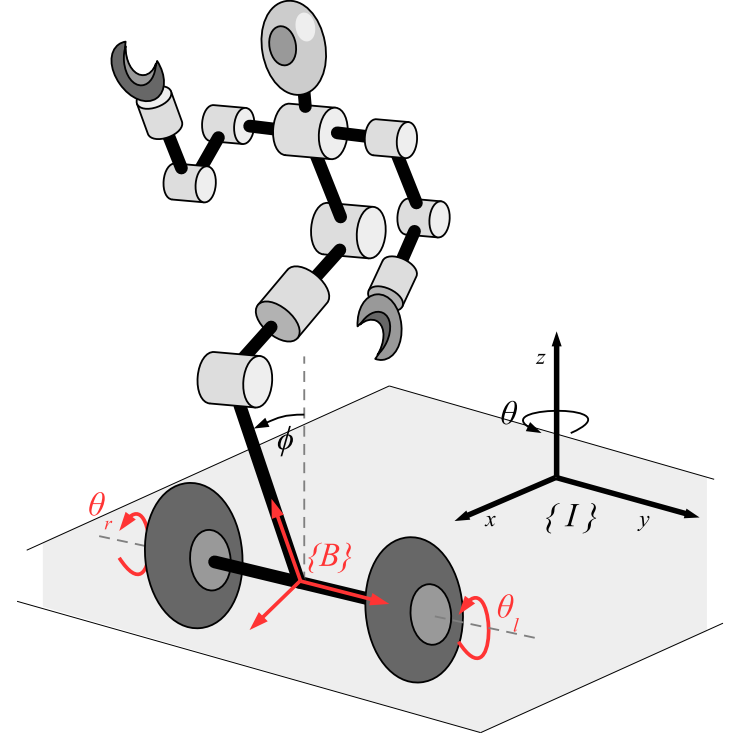

Fig. 2: Generic reference frames for a wheeled humanoid.

of $\{\mathcal{B}\}$ and known the joints configuration, we can define the robot configuration space.

We indicate with $n_{f b}$ the number of independent variables used to describe the position and orientation of the base frame with respect to $\{\mathcal{I}\}$ and we define $q_{f b} \in \mathbb{R}^{n_{f b}}$ the passive freebody linear and angular DoF of the base link.

Let $q_{m p} \in \mathbb{R}^{n_{m p}}$ be the vector which groups the DoF of the mobile platform and $q_{u b} \in \mathbb{R}^{n_{u b}}$ the vector of the actuated upper body joint angles $\left(n=n_{m p}+n_{u b}\right)$.

The Lagrangian coordinates needed to describe the robot configuration and the generalized velocities are given by

$$
q=\left[\begin{array}{c}
q_{f b} \\
q_{m p} \\
q_{u b}
\end{array}\right], \quad \dot{q}=\left[\begin{array}{c}
\dot{q}_{f b} \\
\dot{q}_{m p} \\
\dot{q}_{u b}
\end{array}\right], \quad q, \dot{q} \in \mathbb{R}^{n+n_{f b}} .
$$

In particular, we take the origin of $\{\mathcal{B}\}$ at the centre of the wheels' axle, as shown in Fig. 2 .

Moreover, we assume that the $z$-position of $\{\mathcal{B}\}$ does not change and that the roll angle is equal to zero. Therefore, we consider $n_{f b}=4$ and $q_{f b}=\left[\begin{array}{llll}x & y & \theta & \phi\end{array}\right]^{T} \in \mathbb{R}^{n_{f b}}$, where $x$ and $y$ describe the base link position on the ground, $\theta$ is the yaw angle and $\phi$ is the pitch angle of the robot.

Regarding the mobile platform, that is composed by two wheels, we indicate with $\theta_{l}$ and $\theta_{r}$ the yaw angles of the left wheel and the right one. Therefore, $n_{m p}=2$ and $q_{m p}=\left[\begin{array}{ll}\theta_{l} & \theta_{r}\end{array}\right]^{T} \in \mathbb{R}^{n_{m p}}$.

\section{B. Robot dynamics}

A wheeled robot is characterized by pure rolling, nonholonomic constraints for each wheel, described by

$$
J_{c}(q) \dot{q}=0
$$

where, considering $n_{c}$ the number of independent equations of the constraint, $J_{c} \in \mathbb{R}^{n_{c} \times\left(n+n_{f b}\right)}$ is the so-called Pfaffian matrix. 
The dynamic equation in the standard form used for constrained systems is then given by

$$
\left\{\begin{array}{l}
M(q) \ddot{q}+C(q, \dot{q}) \dot{q}+G(q)+J_{c}^{T}(q) \lambda=U \tau+J_{f}(q)^{T} f \\
\dot{J}_{c} \dot{q}+J_{c} \ddot{q}=0
\end{array}\right.
$$

where

- $M(q) \in \mathbb{R}^{\left(n_{f b}+n\right) \times\left(n_{f b}+n\right)}$ is the inertia matrix;

- $C(q, \dot{q}) \dot{q}+G(q)=c(q, \dot{q}) \in \mathbb{R}^{n_{f b}+n}$ is the generalized force vector containing the Coriolis, centrifugal and gravity terms;

- $\lambda \in \mathbb{R}^{n_{c}}$ is a vector of Lagrange multipliers;

- $\tau \in \mathbb{R}^{n}$ is the vector of actuated joints torques and $U=$ $\left[\begin{array}{ll}0_{n \times n_{f b}} & \mathbf{I}_{n}\end{array}\right]^{T}$ is the matrix that maps these torques to the space of generalized forces;

- $f \in \mathbb{R}^{n_{f}}$ is the vector of external forces and $J_{f}(q) \in$ $\mathbb{R}^{n_{f} \times\left(n_{f b}+n\right)}$ is the kinematic jacobian corresponding to the point of application of the external forces.

As mentioned above, to find the Pfaffian matrix, we consider the pure rolling constraint for each wheel. This constraint requires that the point of the wheel in contact with the ground is instantaneously at rest. This translates into

$$
\begin{gathered}
\omega_{l} \times\left[\begin{array}{lll}
0 & 0 & -r
\end{array}\right]^{T}+v_{l}=0 \\
\omega_{r} \times\left[\begin{array}{lll}
0 & 0 & -r
\end{array}\right]^{T}+v_{r}=0,
\end{gathered}
$$

where $r$ is the wheel radius, $w_{l}$ and $w_{r}$ are the angular velocities of the left and right wheel and $v_{l}$ and $v_{r}$ are the linear velocities of the centre of the left and right wheel, all expressed in the inertial frame $\{\mathcal{I}\}$. From (1) six equations are obtained: the ones relative to the vertical velocity of the contact point are zero under all conditions, and the four remaining equations can be written in matrix form as

$$
A(q)\left[\begin{array}{ll}
\dot{q}_{f b}^{T} & \dot{q}_{m p}^{T}
\end{array}\right]^{T}=0,
$$

where $A(q) \in \mathbb{R}^{4 \times\left(n_{f b}+n_{m p}\right)}$ and is equal to:

$$
A(q)=\left[\begin{array}{cccccc}
1 & 0 & -a c_{\theta} & -r c_{\theta} & -r c_{\theta} & 0 \\
0 & 1 & -a s_{\theta} & -r s_{\theta} & -r s_{\theta} & 0 \\
1 & 0 & a c_{\theta} & -r c_{\theta} & 0 & -r c_{\theta} \\
0 & 1 & a s_{\theta} & -r s_{\theta} & 0 & -r s_{\theta}
\end{array}\right]
$$

with $a$ the semilength of the axle that connects the two wheels, $c_{\theta}=\cos (\theta)$ and $s_{\theta}=\sin (\theta)$. Since the rank of this matrix is three, $n_{c}=3$ and, indicating with $\tilde{A}(q)$ the matrix composed by the first three rows of $A(q)$, the Pfaffian matrix becomes

$$
J_{c}=\left[\begin{array}{ll}
\tilde{A}(q) & 0_{n_{c} \times n_{u b}}
\end{array}\right] .
$$

\section{WHOLE-BODY DYNAMIC CONTROL}

The proposed control law computes the actuation torques $\tau$ so that the robot accelerations $\ddot{q}$ are equal to some desired, constraints-consistent, accelerations $\ddot{q}_{d}$.

To achieve this, let $\nu \in \mathbb{R}^{n+n_{f b}-n_{c}}$ be the quasi-velocity vector, i.e. a vector of velocities such that

$$
\dot{q}=S(q) \nu
$$

where $S(q) \in \mathbb{R}^{\left(n+n_{f b}\right) \times\left(n+n_{f b}-n_{c}\right)}$ is a linear operator that satisfies the relation

$$
J_{c}(q) S(q)=0 .
$$

We define the following quasi-velocity vector $\nu=\left[\begin{array}{cccc}v & \dot{\theta} & \dot{\phi} & \dot{q}_{u b}^{T}\end{array}\right]^{T}$, where $v \in \mathbb{R}$ is the forward velocity of the base link. Therefore, if we indicate with $S_{f b}(q) \in \mathbb{R}^{\left(n_{f b}+n_{m p}\right) \times n_{c}}$ the matrix that verifies the relation

$$
\left[\begin{array}{c}
\dot{q}_{f b} \\
\dot{q}_{m p}
\end{array}\right]=S_{f b}(q)\left[\begin{array}{c}
v \\
\dot{\theta} \\
\dot{\phi}
\end{array}\right],
$$

the linear operator $S(q)$ can be chosen as

$$
S(q)=\left[\begin{array}{cc}
S_{f b}(q) & 0_{\left(n_{f b}+n_{m p}\right) \times n_{u b}} \\
0_{n_{u b} \times n_{c}} & \mathbf{I}_{n_{u b}}
\end{array}\right] .
$$

Considering the definition of forward velocity and the presence of an axle between the wheels that allows to determine a relation between the angles of the base link and the yaw angles of the wheels, the matrix $S_{f b}(q)$ is equal to

$$
S_{f b}(q)=\left[\begin{array}{ccc}
c_{\theta} & 0 & 0 \\
s_{\theta} & 0 & 0 \\
0 & 1 & 0 \\
0 & 0 & 1 \\
1 / r & -a / r & -1 \\
1 / r & a / r & -1
\end{array}\right] .
$$

Moreover, we indicate with $\nu^{d}$ the desired quasi-velocity vector and with $\dot{\nu}^{d}$ and $\int \nu^{d}$ respectively the derivative and the integral of $\nu^{d}$, given as inputs to a computed torque control law.

To find this law, we apply (2) to the dynamic model equation

$$
M(q) \ddot{q}+C(q, \dot{q}) \dot{q}+G(q)=U \tau-J_{c}^{T}(q) \lambda+J_{f}(q)^{T} f
$$

left multiplying it for $S(q)^{T}$, as in [8], and considering (3) and $n_{f}=0$, i.e. $J_{f}(q)^{T} f=0$, the constrained dynamics becomes

$$
\tilde{M}(q) \dot{\nu}+\tilde{c}(q, \dot{q}, \nu)=\tilde{U} \tau,
$$

where:

$$
\begin{aligned}
& \tilde{M}(q)=S^{T}(q) M(q) S(q) \\
& \tilde{c}(q, \dot{q}, \nu)=S^{T}(q)(M(q) \dot{S}(q, \dot{q}) \nu+C(q, \dot{q}) S(q) \nu+G(q)) \\
& \tilde{U}=S^{T}(q) U .
\end{aligned}
$$

Before designing the control law for the constrained system (5), two assumptions have to be done:

- in VI-A we discuss the coupling between the pitch $\phi$ and the forward displacement $q_{\text {forw }}$, which allows the system to be controlled in its upward position, despite being underactuated;

- in VI-B we show how for this system the integrability of the quasi-velocities holds, which is not true in general for constrained mechanichal systems.

Once these two conditions have been verified, we illustrate the control design idea in the following subsection. 


\section{A. Control design}

At this point, the control problem reduces to choose the generalized torques $\tau$ that satisfy the following relation

$$
\dot{\nu}^{d}-\dot{\nu}+K_{d}\left(\nu^{d}-\nu\right)+K_{p} \int_{0}^{t}\left(\nu^{d}-\nu\right)=0,
$$

where the desired quasi-velocities and their time integral and derivatives are chosen to stabilize the system around the upward position, i.e., $\phi^{d}=0$, and any constant $q_{\text {forw }}^{d}$. Equation (6) can be rewritten as

$$
\dot{\nu}=\dot{\nu}^{d}+K_{d}\left(\nu^{d}-\nu\right)+K_{p} \int_{0}^{t}\left(\nu^{d}-\nu\right),
$$

whose dynamics can be made exponentially stable with a proper choice of the gain matrices $K_{p}$ and $K_{d}$ positive definite.

Applying this to (5), we obtain the following computed torque control law:

$$
\tilde{\tau}=\tilde{U} \tau=\tilde{M}\left(\dot{\nu}^{d}+K_{d}\left(\nu^{d}-\nu\right)+K_{p} \int_{0}^{t}\left(\nu^{d}-\nu\right)\right)+\tilde{c} .
$$

Notice that, with the selected quasi-velocities vector, the matrix $\tilde{U} \in \mathbb{R}^{\left(n_{f b}+n-n_{c}\right) \times n}$ is full column rank and equal to

$$
\tilde{U}=S(q)^{T} U=\left[\begin{array}{cc}
\tilde{U}_{f b} & 0_{n_{c} \times n_{u b}} \\
0_{n_{u b} \times n_{m p}} & \mathbf{I}_{n_{u b}}
\end{array}\right],
$$

where $\tilde{U}_{f b} \in \mathbb{R}^{n_{c} \times n_{m p}}$ is given by

$$
\tilde{U}_{f b}=\left[\begin{array}{cc}
1 / r & 1 / r \\
-a / r & a / r \\
-1 & -1
\end{array}\right] .
$$

To find the actuated joints torques, since the first and the third rows of $\tilde{U}$ are linearly dependent, the first row is deleted and, indicating with $\tilde{U}_{s}$ the resulting submatrix, one has

$$
\tau=\tilde{U}_{s}^{-1} \tilde{\tau}_{s},
$$

where $\tilde{\tau}_{s} \in \mathbb{R}^{n}$ is the vector obtained neglecting the first element of $\tilde{\tau}$, corresponding to the generalized forces acting on the forward velocity.

\section{EXPERIMENTAL RESULTS}

In this section we report on experimental results for the proposed control method applied to the two-wheeled mobile robot depicted in Fig. 3, namely ALTER-EGO. It is an underactuated robot with two arms for a total of $n_{u b}=10$ DoFs. Each arm is equipped with variable stiffness actuators [15]. For the purpose of these experiments, the actuators are controlled with a high level of stiffness and can be considered rigid without compromising the validity of the results. For a complete description of the platform see [16].

All experiments have been performed on a flat, non-slippery, surface. We assume that the full robot state information is available, which is obtained thanks to a state estimator which is beyond the scope of this paper. The control law of the robot has been implemented in C++ using ROS middleware. The sample rate for the controller is $T_{s}=0.0025 \mathrm{~s}$.

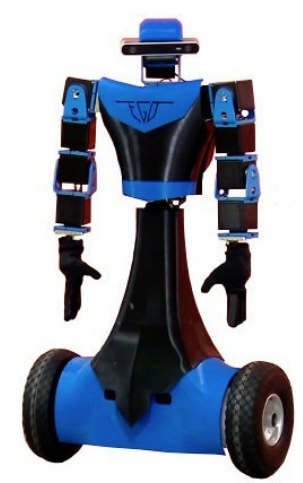

(a)

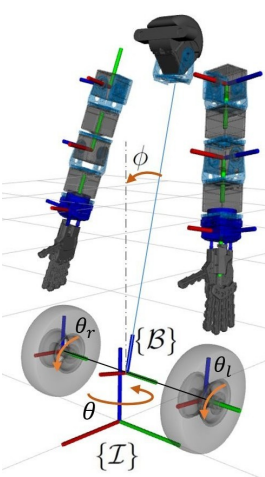

(b)
Fig. 3: Experimental setup: the robot ALTER-EGO (a), and its model reporting relevant reference frames (b).

It is worth noting that no disturbance estimator has been used, and that the application point of the perturbation in the different experiments was different.

The disturbances were applied by hand and not in a repeatable manner, and in this sense the reported results are to be considered preliminary yet encouraging.

The gain matrices $K_{d}$ and $K_{p}$ have been selected with high diagonal values (between 100 and 320 on all coordinates), except for the linear displacement which was given a value of 30. To do this, an experimental tuning has been done: at first, we tuned the $K_{p}$ and $K_{d}$ gain relative to the pitch angle, increasing them until the robot oscillates in resonance. Then, once the resonance condition has been reached, we added a damping contribution by increasing the linear velocity gain (the first element of $K_{d}$ ) until the robot is in the upward position without oscillating. In the end, we tuned the others diagonal values to nullify the errors on the relative Lagrangian coordinates and generalized velocities. Moreover, to fully exploit the whole-body capabilities of the proposed method, extra-diagonal terms were used in the gains to stress the coupling between shoulder and pitch dynamics.

\section{A. Experiments}

1) Static disturbances: first, we tested the control system by loading the robot with a series of increasing weights to generate a static disturbance effect. The results are depicted in Fig. 4a to 4e The constraint equations (represented by the value of $J_{c}(q) \dot{q}$ rows) remain limited during the whole evolution, while the error on the pitch angle remains limited and reaches constant values for increasing payloads.

2) Dynamic disturbance (shoulder): to test for dynamic disturbances, we pushed the robot at the shoulder level and observed its reaction, see Fig. 5 The effect of the perturbation is visible in its pitch angle error diagram. The constraint equations remain limited during the experiment. In particular, as evident from the attached video, the upper body motion of the arms contributes visibly to the stabilization of the pitch angle. Once the pitch angle is stabilized, the robot slowly goes back to its initial sagittal displacement. 


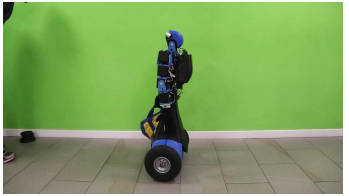

(a) $1.5 \mathrm{~kg}$

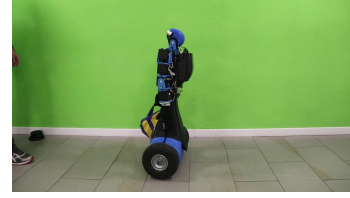

(b) $2.5 \mathrm{~kg}$

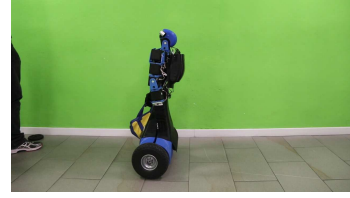

(c) $3.5 \mathrm{~kg}$

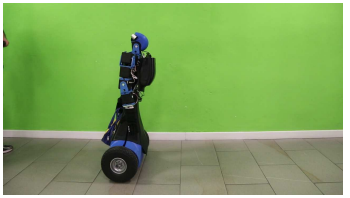

(d) $4.5 \mathrm{~kg}$

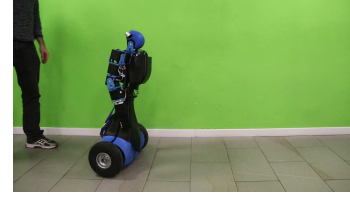

(e) $6.5 \mathrm{Kg}$

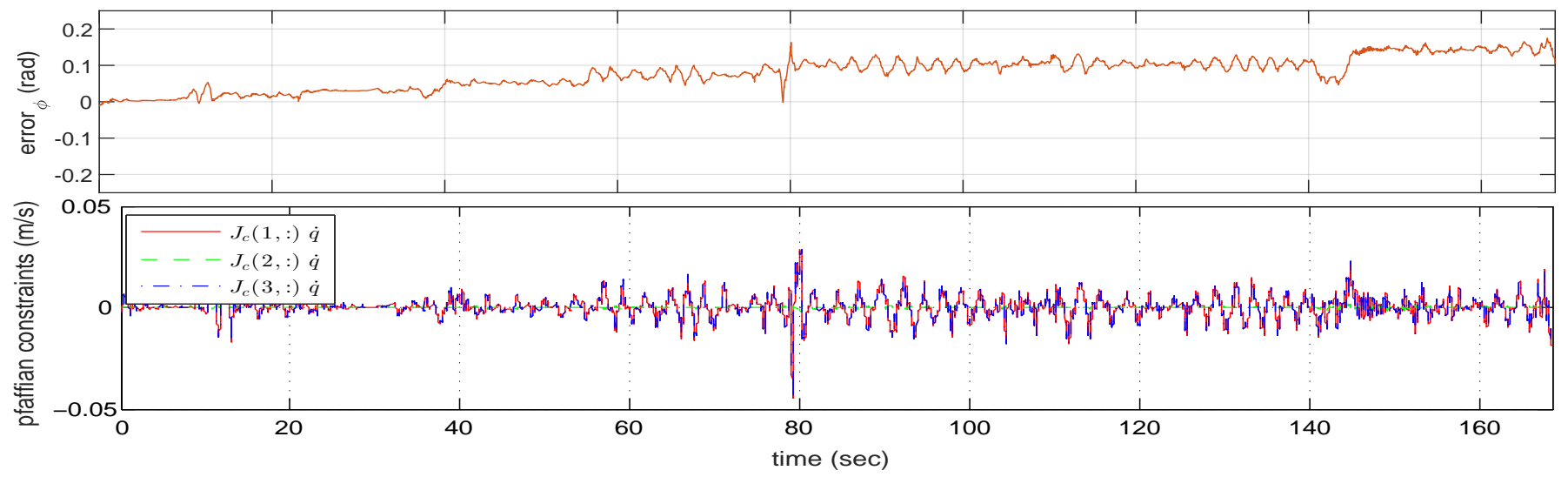

Fig. 4: Experiment 1 - Static disturbances.
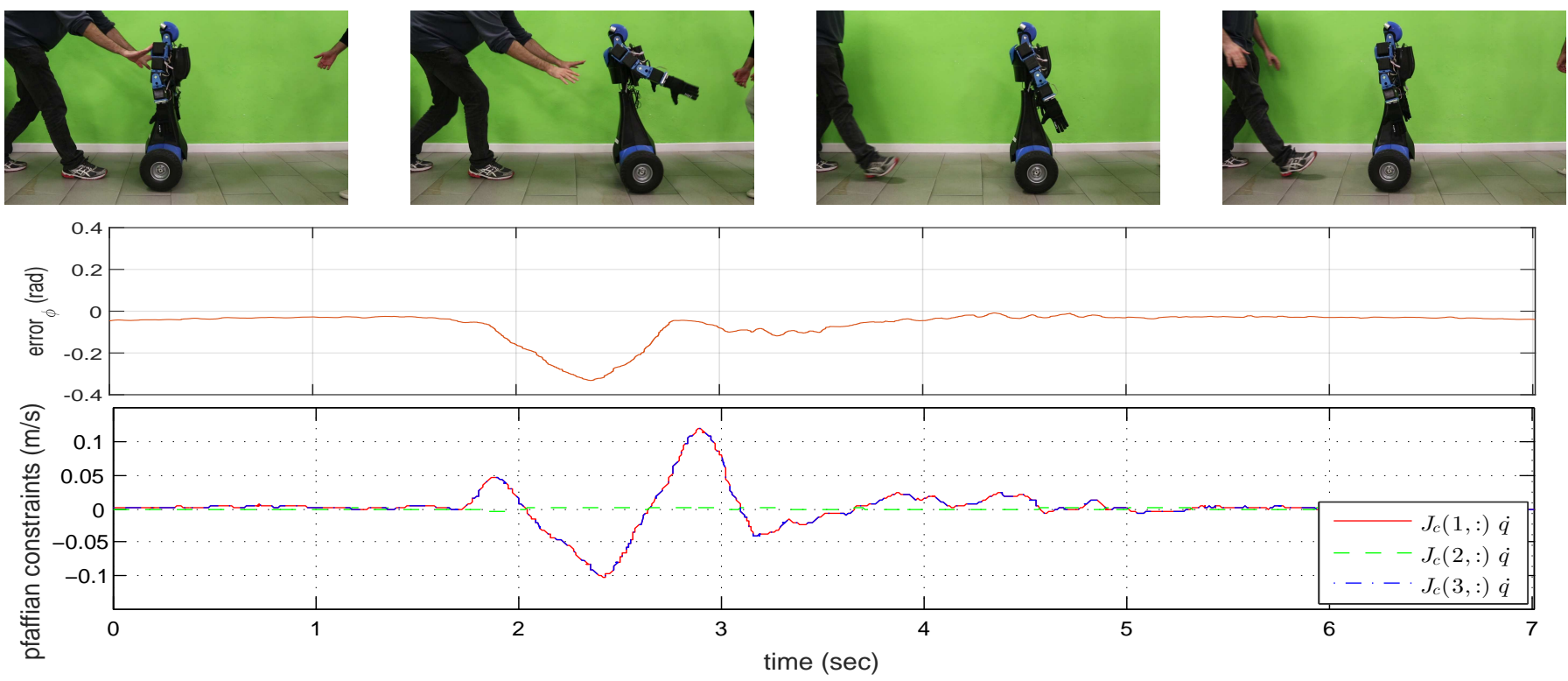

Fig. 5: Experiment 2 - Dynamic disturbance (shoulder).

3) Dynamic disturbance (arms): we applied disturbances to the robot by holding one or both of its hands and pushing them, see Fig. 6. The pitch angle displacement is reported alongside with the constraint equation.

4) Interaction with the environment (opening/closing of a drawer): in this experiment the robot references are calculated with a task-priority approach [17] using a second order kinematic control law on the quasi-velocities, considering the following priority hierarchy: base-link, pitch and yaw at the same priority, upper-body motion at a lower priority. Results are reported in Fig. 7. In particular, the constraint magnitude remains limited, slightly increasing during the initial and middle phase of the task, corresponding to the moments when the robot has to overcome the static friction force of the drawer.

In all these preliminary experiments, despite the unmodeled dynamics in the robot arms, the results were satisfactory. Further investigations will be conducted with a more precise stimulus of disturbance of the robot in order to quantify the entity of the disturbances that can be applied.

\section{B. Methods Comparison}

We compared our method with the traditional approach in which the balancing is ensured by the mobile base only [8]. In the comparison we report the mobile base is commanded through a LQR control. Simulations have been conducted applying a disturbance on the shoulders of the robot, as done in IV-A2, 

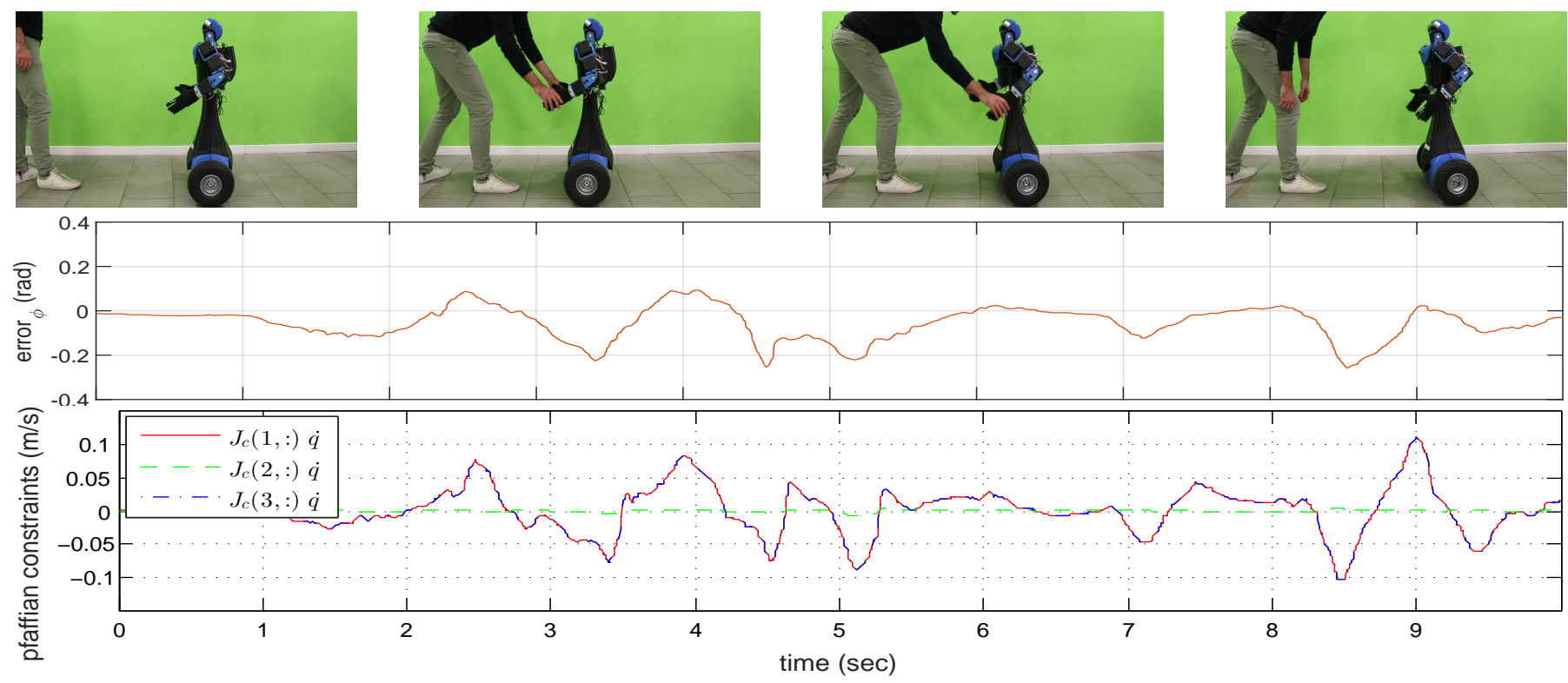

Fig. 6: Experiment 3 - Dynamic disturbance (arms).

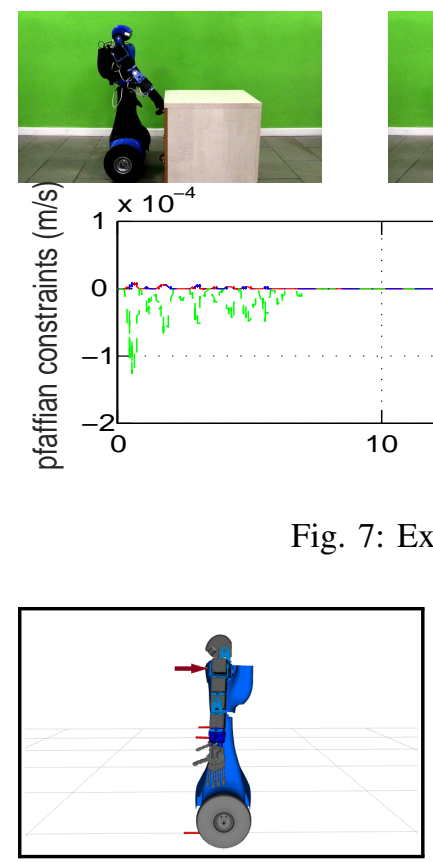

(a) $\mathrm{T}=2.5 \mathrm{~s}$
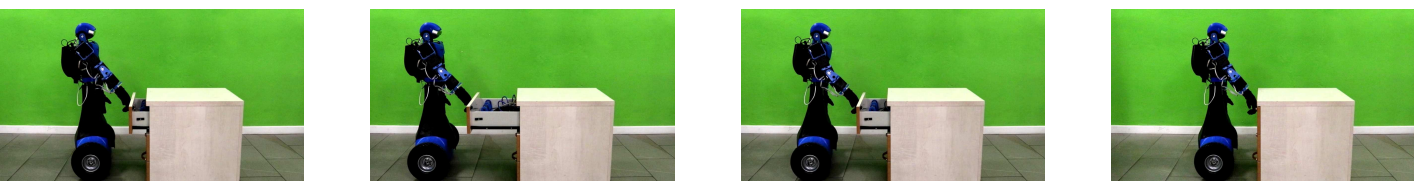

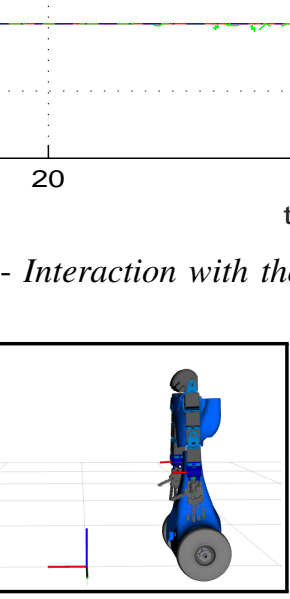

(b) $\mathrm{T}=3.645 \mathrm{~s}$

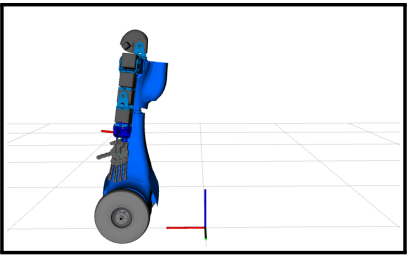

(c) $\mathrm{T}=5.464 \mathrm{~s}$

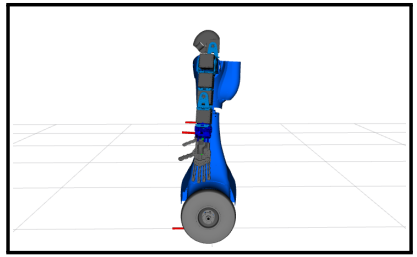

(d) $\mathrm{T}=26.2889 \mathrm{~s}$

(e) LQR Control $\left(Q_{q_{\text {forw }}}=1.06 \cdot 10^{7}\right)$

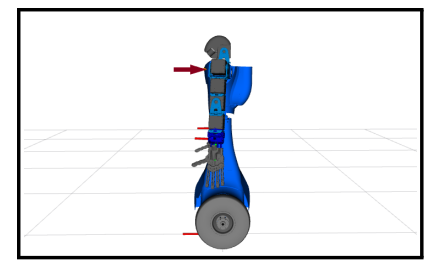

(f) $\mathrm{T}=2.5 \mathrm{~s}$

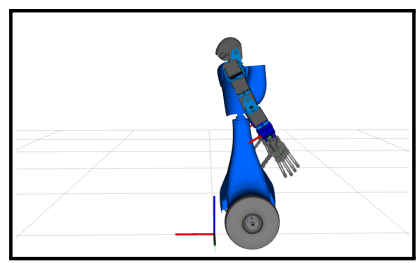

(g) $\mathrm{T}=3.122 \mathrm{~s}$

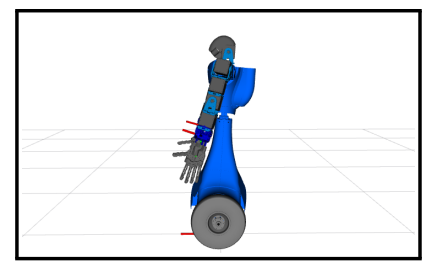

(h) $\mathrm{T}=3.803 \mathrm{~s}$

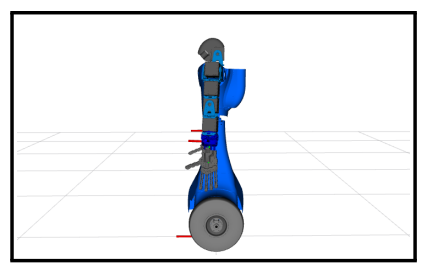

(i) $\mathrm{T}=5.9281 \mathrm{~s}$

(j) Dynamic Whole-Body Control

Fig. 8: LQR Control vs Dynamic Whole-Body Control (DWBC), simulation results. 
In particular, we apply a force of $-50 \mathrm{~N}$ for $0.025 s$ on each shoulder along the $x$-axis of the base frame (Fig. $8 \mathrm{f}$ and Fig. 8a). For this reason, we choose to compare the proposed method with a LQR control, designed on the mobile base dynamics and where the $\mathrm{Q}$ element related to the $q_{\text {forw }}$ variable $\left(Q_{q_{\text {forv }}}\right)$ changes. In particular, if we indicate with $x=\left[\begin{array}{llllll}q_{\text {forw }} & \theta & \phi & v & \dot{\theta} & \dot{\phi}\end{array}\right]^{T}$ the state vector, the chosen

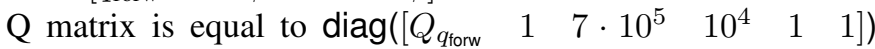
and, if we indicate with $u=\tau_{m p}$, the $\mathrm{R}$ matrix is taken equal to $0.15 \mathbf{I}_{n_{m p}}$.

The difference between the two approaches are already visible from Fig. 8 The advantages introduced with the new approach can be shown with the analysis the $q_{\text {forw }}$ error, the settling time and the torques commanded at the wheels.

Therefore, indicating with $e_{q_{\text {forw }}}$ the vector composed by the $q_{\text {forw }}$ error of each simulation step, the Euclidean norm of $e_{q_{\text {forw }}}$ obtained with the proposed method, that is equal to $4.0604 \mathrm{~m}$, is smaller than the one obtained with the LQR, as shown in Fig. 9

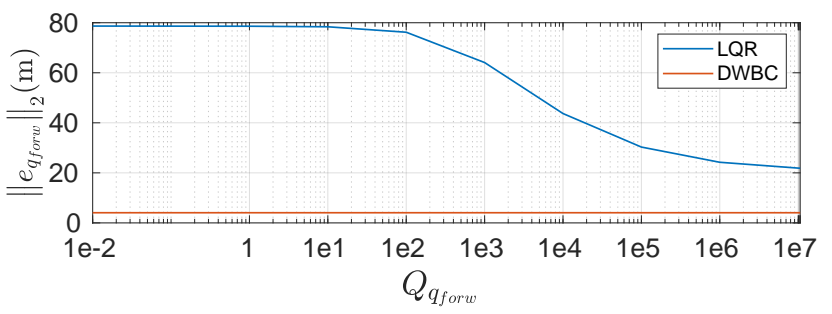

Fig. 9: Comparison between $\left\|e_{q_{\text {forw }}}\right\|_{2}$ obtained with the LQR control and the one obtained with DWBC

The settling time of our approach is better than the one obtained with the LQR control, as we can already see in Fig. $8 \mathrm{i}$ and Fig. $8 \mathrm{~d}$; in our case $t=3.4281 \mathrm{~s}$ that is much lower than $t=23.7889 \mathrm{~s}$ that is the minimum value obtained with the LQR method.

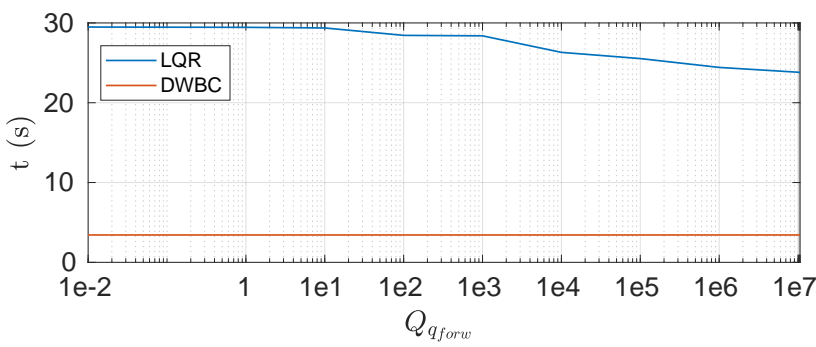

Fig. 10: Comparison between the settling time $t$ obtained with the LQR control and the one obtained with DWBC

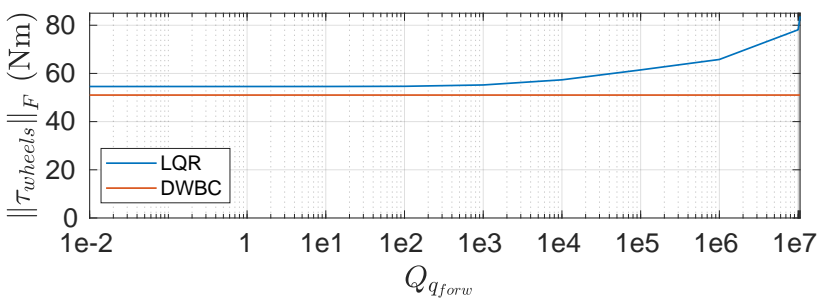

Fig. 11: Comparison between $\left\|\tau_{\text {wheels }}\right\|_{F}$ obtained with the LQR control and the one obtained with DWBC
However, if we indicate with $\tau_{\text {wheels }}$ the matrix obtained stacking at each simulation step the vector $\left[\begin{array}{ll}\tau_{\theta_{l}} & \tau_{\theta_{r}}\end{array}\right]$, also the Frobenius norm of $\tau_{\text {wheels }}$ obtained with our control approach $(51.0108 \mathrm{Nm}$ ) is smaller than the one of the LQR, (Fig. 11).

Therefore, with this comparison, we can conclude that the performances of the proposed method, relative to $q_{\text {forw }}$ error and the settling time, are superior than the ones reached with the LQR traditional method. Moreover, since DWBC requires less wheel torques, it could reject disturbances of higher magnitude than the ones tolerated by LQR.

In the attached video we also added a qualitative comparison between LQR and DWBC, where it is evident the wholebody behavior of the proposed method. Due to the difficulty of replicating the same disturbance applied to the robot, an exhaustive experimental comparison will be addressed in the future.

\section{CONCLUSIONS}

In this work we proposed a new method for whole-body nonlinear control of wheeled humanoids. We derive a control law for the constrained system as a computed torque in the quasi-velocities. In the derivation, we show how two important properties hold for this kind of robot, namely strong inertial coupling between wheeled base and pitch dynamics and the integrability of quasi-velocities when considering kinematic unicycle constraints and flat motion. We illustrate the idea behind the control design and show its effectiveness in several experiments.

Future work will be devoted to a theoretical investigation of the stability of the closed loop system and to the application of the proposed method to several static and dynamic tasks. Learning of feedback gains and feed-forward references to perform complex tasks in daily environments is of interest, as well as the extension to other robotic platforms such as legged ones. Moreover, a thorough analysis of the sampling time will be performed, to correctly estimate the minimum sampling time requested for the considered applications. Finally, to enable experimental comparison with other control approaches, we will consider to exploit benchmarking methods for robotics applications (e.g. project Disturbance funded under the project framework EUROBENCH [18]).

\section{APPENDIX}

\section{A. Controllability of the underactuated system}

When designing a control law for this kind of system a challenge arises due to the need of controlling two variables (pitch angle and forward velocity) with a single input (sum of wheel torques). We can overcome this issue thanks to the fact that the property of strong inertial coupling [19] holds in an interval around the upward position. This can be easily seen considering the planar dynamics of the robot which is similar to the one of a cart-pole system. In particular, the coupling term in the inertia matrix is given by

$$
M_{\ddot{\phi}, \dot{v}}=\hat{M} L \cos \phi-\hat{m} r-\frac{4 k^{2} J_{m}}{r}
$$

where $\hat{M}$ is the total mass of the robot, $\hat{m}$ is the wheel mass, $L$ is the position of the centre of mass, $k$ is the 
inverse of the gear ratio, $J_{m}$ is the motor inertia. To guarantee strong inertial coupling between $\phi$ and $q_{\text {forw }}$ it suffices to have $M_{\ddot{\phi}, \dot{v}} \neq 0$, which is true for $\phi \in(-p, p)$, with $p=\arccos \left(\frac{\hat{m} r}{\hat{M} L}+\frac{4 k^{2} J_{m}}{r \hat{M} L}\right)$ that, for the robot described in Section IV, has a value of around $88^{\circ}$. Therefore, it is possible to stabilize the robot around the point $\phi=0$, the robot upward position (Fig. 2), by means of partial feedback linearization or LQR control, or designing a new control law inspired by composite feedback for singularly perturbed systems [20], where the input variable is composed by two components: one responsible for stabilizing slow dynamics of the system around a reduced equilibrium manifold, and one responsible for stabilizing fast dynamics around the slow, reduced manifold. Composite feedback approach was previously applied to the control of lightweight flexible manipulators in [21].

\section{B. Integrability of quasi-velocities}

The quasi-velocities cannot be integrated unless a one-toone correspondence between $\int \nu$ and $q$ exists ([22]) and, if it does exist, $\int \nu$ is called quasi-coordinates vector. Therefore, to demonstrate the integrability of quasi-velocities, it must be proved that it is possible to write $\int \nu$ as an explicit function of $q$. In this case of study, to do this, it is necessary to consider a subset of $\dot{q}$, that is denoted with $\dot{q}_{r e d} \in \mathbb{R}^{n_{f b}+n-n_{c}}$ and is equal to:

$$
\dot{q}_{\text {red }}=\left[\begin{array}{c}
\dot{\phi} \\
\dot{q}_{m p} \\
\dot{q}_{u b}
\end{array}\right]=S_{\text {red }} \nu,
$$

where $S_{\text {red }} \in \mathbb{R}^{\left(n_{f b}+n-n_{c}\right) \times\left(n_{f b}+n-n_{c}\right)}$ is obtained by removing the first three rows from $S(q)$ given in (4):

$$
S_{\text {red }}=\left[\begin{array}{cc}
S_{f b_{\text {red }}} & 0_{n_{c} \times n_{u b}} \\
0_{n_{u b} \times n_{c}} & \mathbf{I}_{n_{u b}}
\end{array}\right],
$$

with

$$
S_{f b_{\text {red }}}=\left[\begin{array}{ccc}
0 & 0 & 1 \\
1 / r & -a / r & -1 \\
1 / r & a / r & -1
\end{array}\right] .
$$

As it can be seen, $S_{r e d}$ is a square, constant matrix. Its inverse exists and it is equal to:

$$
S_{r e d}^{-1}=\left[\begin{array}{cc}
S_{f b_{r e d}}^{-1} & 0_{n_{c} \times n_{u b}} \\
0_{n_{u b} \times n_{c}} & \mathbf{I}_{n_{u b}}
\end{array}\right],
$$

with

$$
S_{f b_{\text {red }}}^{-1}=\left[\begin{array}{ccc}
r & r / 2 & r / 2 \\
0 & -r /(2 a) & r /(2 a) \\
1 & 0 & 0
\end{array}\right] .
$$

Therefore, it is possible to invert 8 and, as a result, it is possible to compute $\int \nu$ as

$$
\int \nu=S_{\text {red }}^{-1} \int \dot{q}_{\text {red }}=S_{\text {red }}^{-1} q_{\text {red }} .
$$

In conclusion, in this case of study it is possible to integrate the quasi-velocity vector since it can always be expressed as an explicit function of a subset of the joint variables. It is worth noting that, thanks to this property, a static feedback on the measured quasi-coordinates can be applied.

\section{REFERENCES}

[1] Ronald Ping Man Chan, Karl A Stol, and C Roger Halkyard. Review of modelling and control of two-wheeled robots. Annual reviews in control, 37(1):89-103, 2013.

[2] Umashankar Nagarajan, George Kantor, and Ralph Hollis. The ballbot: An omnidirectional balancing mobile robot. The International Journal of Robotics Research, 33(6):917-930, 2014.

[3] Marc H Raibert. Legged robots that balance. MIT press, 1986.

[4] Alexander Dietrich. Whole-Body Impedance Control of Wheeled Humanoid Robots, volume 116. Springer, 2016.

[5] E. Farnioli, M. Gabiccini, and A. Bicchi. Toward whole-body locomanipulation: Experimental results on multi-contact interaction with the walk-man robot. In 2016 IEEE/RSJ International Conference on Intelligent Robots and Systems (IROS), pages 1372-1379, Oct 2016.

[6] Bernd Henze, Máximo A Roa, and Christian Ott. Passivity-based wholebody balancing for torque-controlled humanoid robots in multi-contact scenarios. The International Journal of Robotics Research, 35(12):15221543, 2016.

[7] Martin Lakie, Nicholas Caplan, and Ian D Loram. Human balancing of an inverted pendulum with a compliant linkage: neural control by anticipatory intermittent bias. The Journal of physiology, 551(1):357370, 2003.

[8] Seonghee Jeong and Takayuki Takahashi. Wheeled inverted pendulum type assistant robot: design concept and mobile control. Intelligent Service Robotics, 1(4):313-320, 2008.

[9] Mark W Spong. Partial feedback linearization of underactuated mechanical systems. In Intelligent Robots and Systems' 94.'Advanced Robotic Systems and the Real World', IROS'94. Proceedings of the IEEE/RSJ/GI International Conference on, volume 1, pages 314-321. IEEE, 1994.

[10] Tao Feng, Tao Liu, Xu Wang, Zhao Xu, Meng Zhang, and Shengchao Han. Modeling and implementation of two-wheel self-balancing robot equipped with supporting arms. In 2011 6th IEEE Conference on Industrial Electronics and Applications, pages 713-718. IEEE, 2011.

[11] Scott R Kuindersma, Edward Hannigan, Dirk Ruiken, and Roderic A Grupen. Dexterous mobility with the ubot-5 mobile manipulator. In 2009 International Conference on Advanced Robotics, pages 1-7. IEEE, 2009.

[12] Munzir Zafar, Seth Hutchinson, and Evangelos A Theodorou. Hierarchical optimization for whole-body control of wheeled inverted pendulum humanoids. arXiv preprint arXiv:1810.03074, 2018.

[13] Munzir Zafar and Henrik I Christensen. Whole body control of a wheeled inverted pendulum humanoid. In 2016 IEEE-RAS 16th International Conference on Humanoid Robots (Humanoids), pages 8994. IEEE, 2016.

[14] Mike Stilman, Jon Olson, and William Gloss. Golem krang: Dynamically stable humanoid robot for mobile manipulation. In 2010 IEEE International Conference on Robotics and Automation, pages 33043309. IEEE, 2010.

[15] Cosimo Della Santina, Cristina Piazza, Gian Maria Gasparri, Manuel Bonilla, Manuel Giuseppe Catalano, Giorgio Grioli, Manolo Garabini, and Antonio Bicchi. The quest for natural machine motion: An open platform to fast-prototyping articulated soft robots. IEEE Robotics \& Automation Magazine, 24(1):48-56, 2017.

[16] Gianluca Lentini et al. ALTER-EGO: A mobile robot with functionally anthropomorphic upper body designed for physical interaction. IEEE Robotics \& Automation Magazine, 2019.

[17] Bruno Siciliano, Lorenzo Sciavicco, Luigi Villani, and Giuseppe Oriolo. Robotics: modelling, planning and control. Springer Science \& Business Media, 2010.

[18] Eurobench. http://eurobench2020.eu

[19] Mark W Spong. Underactuated mechanical systems. In Control problems in robotics and automation, pages 135-150. Springer, 1998.

[20] Petar Kokotovic, Hassan K Khali, and John O'reilly. Singular perturbation methods in control: analysis and design, volume 25. Siam, 1999.

[21] Bruno Siciliano and Wayne J Book. A singular perturbation approach to control of lightweight flexible manipulators. The International Journal of Robotics Research, 7(4):79-90, 1988.

[22] Farhad Aghili. Dynamics and control of constrained mechanical systems in terms of reduced quasi-velocities. In Robotics and Automation, 2008. ICRA 2008. IEEE International Conference on, pages 1225-1232. IEEE, 2008. 